\title{
The Impact of Obstructive Sleep Apnea on Metabolic and Inflammatory Markers in Consecutive Patients with Metabolic Syndrome
}

\author{
Luciano F. Drager ${ }^{1,2 *}$, Heno F. Lopes ${ }^{1}$, Cristiane Maki-Nunes ${ }^{1}$, Ivani C. Trombetta ${ }^{1}$, Edgar Toschi-Dias ${ }^{1}$, \\ Maria Janieire N. N. Alves ${ }^{1}$, Raffael F. Fraga ${ }^{1}$, Jonathan C. Jun ${ }^{2}$, Carlos E. Negrão ${ }^{1}$, Eduardo M. Krieger ${ }^{1}$, \\ Vsevolod Y. Polotsky ${ }^{2}$, Geraldo Lorenzi-Filho' \\ 1 Heart Institute (InCor), University of São Paulo Medical School, São Paulo, Brazil, 2 Division of Pulmonary and Critical Care Medicine, Johns Hopkins University School of \\ Medicine, Baltimore, Maryland, United States of America
}

\begin{abstract}
Background: Obstructive Sleep Apnea (OSA) is tightly linked to some components of Metabolic Syndrome (MetS). However, most of the evidence evaluated individual components of the MetS or patients with a diagnosis of OSA that were referred for sleep studies due to sleep complaints. Therefore, it is not clear whether OSA exacerbates the metabolic abnormalities in a representative sample of patients with MetS.

Methodology/Principal Findings: We studied 152 consecutive patients (age $48 \pm 9$ years, body mass index $32.3 \pm 3.4 \mathrm{Kg} / \mathrm{m}^{2}$ ) newly diagnosed with MetS (Adult Treatment Panel III). All participants underwent standard polysomnography irrespective of sleep complaints, and laboratory measurements (glucose, lipid profile, uric acid and C-reactive protein). The prevalence of OSA (apnea-hypopnea index $\geq 15$ events per hour of sleep) was $60.5 \%$. Patients with OSA exhibited significantly higher levels of blood pressure, glucose, triglycerides, cholesterol, LDL, cholesterol/HDL ratio, triglycerides/HDL ratio, uric acid and C-reactive protein than patients without OSA. OSA was independently associated with 2 MetS criteria: triglycerides: OR: 3.26 (1.47-7.21) and glucose: OR: 2.31 (1.12-4.80). OSA was also independently associated with increased cholesterol/HDL ratio: OR: 2.38 (1.08-5.24), uric acid: OR: 4.19 (1.70-10.35) and C-reactive protein: OR: 6.10 (2.64-14.11). Indices of sleep apnea severity, apnea-hypopnea index and minimum oxygen saturation, were independently associated with increased levels of triglycerides, glucose as well as cholesterol/HDL ratio, uric acid and C-reactive protein. Excessive daytime sleepiness had no effect on the metabolic and inflammatory parameters.
\end{abstract}

Conclusions/Significance: Unrecognized OSA is common in consecutive patients with MetS. OSA may contribute to metabolic dysregulation and systemic inflammation in patients with MetS, regardless of symptoms of daytime sleepiness.

Citation: Drager LF, Lopes HF, Maki-Nunes C, Trombetta IC, Toschi-Dias E, et al. (2010) The Impact of Obstructive Sleep Apnea on Metabolic and Inflammatory Markers in Consecutive Patients with Metabolic Syndrome. PLoS ONE 5(8): e12065. doi:10.1371/journal.pone.0012065

Editor: Adrian V Hernandez, Lerner Research Institute, United States of America

Received May 27, 2010; Accepted July 14, 2010; Published August 11, 2010

Copyright: (C) 2010 Drager et al. This is an open-access article distributed under the terms of the Creative Commons Attribution License, which permits unrestricted use, distribution, and reproduction in any medium, provided the original author and source are credited.

Funding: The study was supported by the Conselho Nacional de Desenvolvimento Cientifico e Tecnologico (CNPq) and Fundacao Zerbini, Brazil. Dr. Drager is supported by the CNPq (\# 200032/2009-7) and Fundacao Zerbini, Brazil. Dr. Jun is supported by the National Sleep Foundation/American Lung Association Pickwick Grant (SF-78568 N) and National Institutes of Health (NIH) T32 training grant (HL07534). Dr. Polotsky is supported by NIH (R01 HL80105, 5P50HL084945), the American Heart Association (grant 0765293U) and United States Israel Binational Science Foundation (grant BSF No. 2005265). The funders had no role in study design, data collection and analysis, decision to publish, or preparation of the manuscript.

Competing Interests: The authors have declared that no competing interests exist.

*E-mail: luciano.drager@incor.usp.br

\section{Introduction}

Metabolic syndrome (MetS) constitutes a clustering of metabolic and cardiovascular abnormalities including central obesity, insulin resistance, dyslipidemia, and increased blood pressure in the same individual [1]. Despite some controversy, MetS is associated with higher cardiovascular risk than one might expect from simple addition of its individual components [2-4]. However, other factors may contribute to the high cardiovascular burden observed in patients with MetS.

Obstructive sleep apnea (OSA) is characterized by recurrent episodes of partial or complete obstruction of the upper airway, intermittent hypoxia and frequent arousals from sleep [5]. There is abundant evidence from humans and animals suggesting that OSA may impact every aspect of MetS, including obesity [6], hypertension [7], insulin resistance [8] and dyslipidemia $[9,10]$. Furthermore, OSA and MetS have been previously shown to co-exist [11-20]. However, previous studies were limited by small sample size and/or selection bias, because they included patients referred for sleep studies due to sleep-related complaints. Thus, the prevalence and impact of OSA in consecutive patients with MetS have not been adequately explored. It is not certain whether the overlap between OSA and MetS is simply a result of underlying obesity, or if OSA represents an additional burden that exacerbates metabolic dysfunction and systemic inflammation in patients with MetS. The impact of daytime sleepiness on markers of cardiovascular risk in patients with MetS is unknown. 
In the present investigation we enrolled consecutive patients with MetS with no previous diagnosis of OSA in order to evaluate if the presence of OSA is independently associated with (1) parameters of MetS, (2) parameters associated with cardiovascular risk but not included in the MetS definition. Moreover, we explored if effects of OSA on metabolic and inflammatory indices are modulated by the extent of daytime sleepiness.

\section{Materials and Methods}

\section{Ethics Statement}

The local Ethics Committee (Institutional Review Board Heart Institute) approved the protocol, and all participants gave written informed consent.

\section{Patients}

We studied consecutive patients with a recent diagnosis of MetS recruited from the Heart Institute (InCor) from October 2008 to December 2009. All participants were asymptomatic outpatients admitted for routine check-up evaluations. No sleep questionnaire was applied at the time of the recruitment. Patients with established cerebrovascular disease, coronary disease, heart failure, rheumatologic diseases, renal failure; hypothyroidism, pregnancy, history of smoking, and regular exercisers were excluded as well as patients with a previous diagnosis of OSA. In addition, we excluded patients who were using hypoglycemic medications, insulin, fibrates, statins, uricosuric agents (such as allopurinol), steroids and contraceptives. All participants underwent a detailed history and physical. The body mass index was calculated after body weight and height were measured in subjects wearing light clothing without shoes. Waist circumference was measured with soft tape on standing subjects midway between the lowest rib and the iliac crest. Two blood pressure recordings were obtained from the right arm of patients in a sitting position after 15 minutes of rest at 5-minute intervals, and their mean value was calculated. The diagnosis of hypertension was based on current guidelines [21].

\section{Blood samples}

Fasting blood samples were drawn for determination of glucose, total cholesterol, low-density lipoprotein (LDL), high-density lipoprotein (HDL), triglycerides, and uric acid using enzymatic methods. High-sensitivity C-reactive protein was measured by using particle enhanced immunonephelometry (Dade Behring, Inc. Deerfield, Illinois). All samples were collected in the absence of clinical evidence of active infection/ inflammatory processes such as viral infections.

\section{Definition of metabolic syndrome}

MetS was diagnosed according to the National Cholesterol Education Program, Adult Treatment Panel III (NCEP III) (1), if 3 of the 5 following factors were present: 1) waist circumference ( $\geq 102 \mathrm{~cm}$ in men and $\geq 88$ in women), 2) triglycerides $\geq 150 \mathrm{mg} /$ $\mathrm{dL}$, or patient on specific drug treatment, 3) $\mathrm{HDL}<40 \mathrm{mg} / \mathrm{dL}$ in men and $<50 \mathrm{mg} / \mathrm{mg} / \mathrm{dL}$ in women, or when on specific drug treatment, 4) arterial blood pressure $\geq 130$ or $85 \mathrm{~mm} \mathrm{Hg}$ for systolic and diastolic blood pressure, respectively, or patient on antihypertensive drug treatment, and 5) fasting glucose $\geq 100 \mathrm{mg} /$ $\mathrm{dL}$ or patient on specific drug treatment.

\section{Sleep Parameters}

Within 1 month after blood sample collection, all participants underwent a standard overnight polysomnography as previously described [22]. Apnea was defined as complete cessation of airflow for at least 10 seconds, associated with oxygen desaturation of $3 \%$.
Hypopnea was defined as a reduction in respiratory signals for at least 10 seconds associated with oxygen desaturation of $3 \%$. The apnea-hypopnea index (AHI) was calculated as the total number of respiratory events (apneas plus hypopneas) per hour of sleep. The AHI cutoffs for mild, moderate and severe OSA were 5 to $14.9,15$ to 29.9 , and $\geq 30$ events per hour of sleep, respectively. Because of a high expected prevalence of OSA in this population, the presence of OSA was also restricted to the moderate to severe cases, i.e., AHI $\geq 15$ events per hour of sleep as previously described [18]. Daytime somnolence was evaluated by the Epworth sleepiness scale [23], with a score of $>10$ considered excessive daytime sleepiness.

\section{Statistical Analysis}

Data were analyzed with SPSS statistical software version 18.0 (Chicago, Illinois, USA). The comparison of continuous variables between patients with and without OSA was performed using the Student $t$ test or Mann-Whitney test, when appropriate. Categorical variables were expressed by frequency distribution and were compared using the Fisher exact test. Continuous variables with normal distribution were expressed as mean \pm SD. Otherwise, they were presented as median (interquartile range). In order to analyze the relative role of OSA either as a categorical variable (presence or absence of OSA) or as a continuous one (AHI, minimum oxygen saturation during sleep and total sleep time below 90\%) we performed two independent analyses: 1) Univariable and multivariable logistic regression analysis to model the association of each MetS criteria (waist circumference, triglycerides, HDL, blood pressure and glucose) and non-MetS risk factors (cholesterol/HDL ratio $\geq 4.5$ [24], triglycerides/HDL ratio $>3$ [25], G-reactive protein $>3 \mathrm{mg} / \mathrm{L}$ [26] and uric acid $>7 \mathrm{mg} / \mathrm{dL}$ [27]) according to the presence of OSA (adjusted for age, sex, race, body mass index and waist circumference); 2) Multiple linear regression analysis (Stepwise linear regression analysis) to evaluate the relative role of markers of OSA severity with absolute values of components of MetS and metabolic and inflammatory variables not included in the MetS criteria (cholesterol/HDL ratio, uric acid and C-reactive protein). We used a $\mathrm{P}=0.15$ as the critical value for entering/excluding variables in the model. Independent variables were age, sex, race, body mass index, waist circumference and sleep parameters. In all multiple regression analysis we avoided the presence of multicollinearity. In a subgroup of patients with MetS and OSA, we also performed a comparison of the independent variables associated with the presence of OSA according to the presence or absence of excessive daytime sleepiness.

\section{Results}

We initially enrolled 210 consecutive patients with a recently confirmed diagnosis of MetS. The final sample was comprised of 152 patients, because 58 subjects met one or more exclusion criteria (Figure 1). Characteristics of patients including age, sex, body mass index and waist circumference were not different between patients included and excluded from the study ( $\mathrm{P}>0.2$ for all comparisons). Forty participants $(26.3 \%)$ were involved in the previous study evaluating the impact of OSA on markers of atherosclerosis in consecutive patients with MetS [28]. Notably only 7 (3.3\%) were excluded from the initial screening because of a previous diagnosis of OSA. The prevalence of OSA (AHI $\geq 15$ events per hour of sleep) in patients with MetS was $60.5 \%$.

Twenty seven patients $(17.8 \%)$ presented with an AHI $<5$ events per hour of sleep. Thirty three patients (21.7\%) presented with an AHI from 5 to 14.9 events per hour of sleep. Moderate 


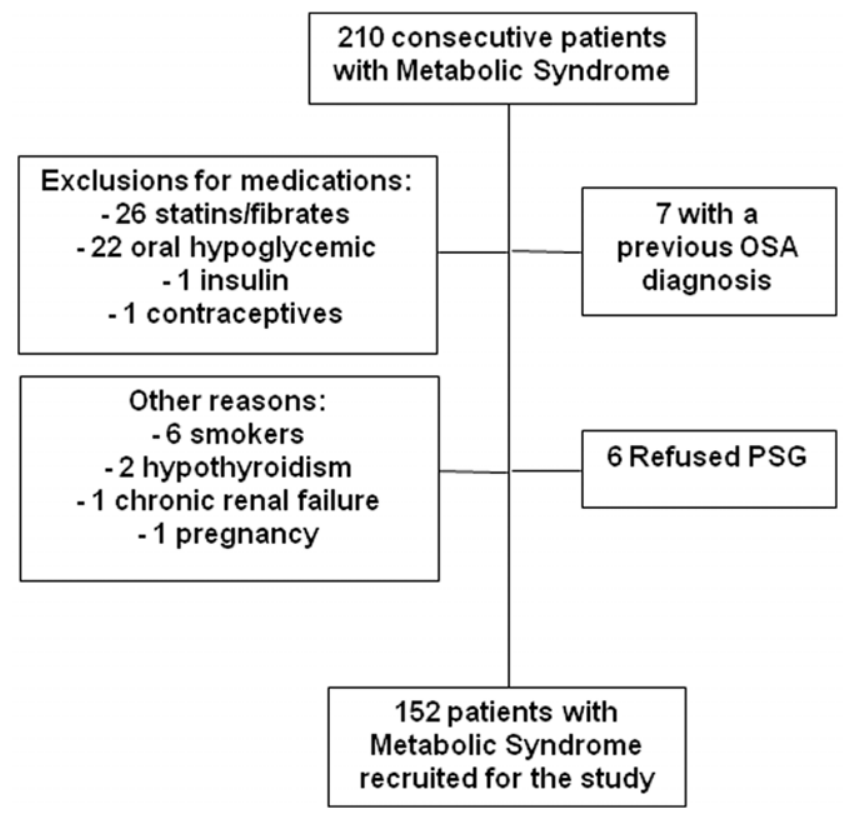

Figure 1. Patient flow diagram. Some patients had multiple exclusions reasons. OSA: Obstructive Sleep Apnea. PSG: Polisomnography.

doi:10.1371/journal.pone.0012065.g001

OSA (AHI 15-29.9 events per hour of sleep) and severe OSA (AHI $\geq 30$ events per hour of sleep) were observed in $34(22.4 \%)$ and 58 patients $(38.1 \%)$, respectively. The demographic, anthropometric and sleep characteristics of the total population of patients studied, as well as comparisons of patients with or without OSA are shown in Table 1. Overall, the present sample included middle age patients. As expected, the great majority of participants were obese with high waist circumference measurements. There were no significant differences in sex, race, body mass index, waist circumference, hypertension, and diabetes status between patients with and without OSA. The anti-hypertensive medications were also similar between hypertensive patients with and without OSA: diuretics $(75 \%$ vs. $72 \%)$, beta-blockers $(38 \%$ vs. $55 \%)$, calcium channel blockers ( $44 \%$ vs. $34 \%$ ), angiotensin-converting enzyme inhibitors $(64 \%$ vs. $50 \%)$ and angiotensin II receptor blockers ( $22 \%$ vs. $17 \%$ ), were similar ( $\mathrm{P}>0.1$ for all comparisons). MetS patients with OSA were older (Table 1). Patients with OSA met a higher number of MetS criteria due to the higher rate of hypertriglyceridemia and hyperglycemia than patients without OSA (Figure 2 and 3). Patients with OSA and MetS had significantly higher levels of MetS-defining parameters including blood pressure, fasting blood glucose and serum triglycerides compared to patients with MetS alone (Table 2). The levels of HDL were similar between patients with and without OSA. In contrast, the levels of several non-MetS parameters including serum total cholesterol, LDL, triglycerides/HDL ratio, cholester$\mathrm{ol} / \mathrm{HDL}$ ratio, uric acid and C-reactive protein were also higher in patients with OSA.

Univariable and multivariable logistic regression analysis (Table 3) showed that the presence of OSA was independently associated with 2 of 5 criteria for MetS (triglycerides and glucose). Moreover, the presence of OSA was independently associated with abnormally elevated cholesterol/HDL ratio, uric acid and C-reactive protein. There was a strong trend for an independent association between the presence of OSA and triglycerides/HDL ratio.

Multiple linear regression analysis showed that the AHI or minimum oxygen saturation during sleep were independently associated with serum levels of triglycerides and glucose as well as with several metabolic and inflammatory parameters not included in the MetS criteria (cholesterol/HDL ratio, uric acid and Creactive protein - Table 4). Although C-reactive protein has a skewed distribution, the residuals from this model were normally distributed.

Systolic and diastolic blood pressure were independently related only to age (data not shown; $\mathrm{P}<0.001$ for both comparisons).

Figure 4 shows that the independent variables associated with OSA (glucose, triglycerides, cholesterol/HDL ratio, uric acid and C-reactive protein) were similar in patients with and without excessive daytime sleepiness.

Table 1. Patient characteristics.

\begin{tabular}{|c|c|c|c|c|}
\hline & Total Sample $(n=152)$ & No OSA $(n=60)$ & OSA $(n=92)$ & $P$ value* \\
\hline Age (years) & $48 \pm 9$ & $46 \pm 8$ & $49 \pm 8$ & 0.02 \\
\hline Males (\%) & 62.5 & 55 & 67 & 0.13 \\
\hline Caucasians (\%) & 74 & 70 & 77 & 0.34 \\
\hline Body mass index $\left(\mathrm{kg} / \mathrm{m}^{2}\right)$ & $32.3 \pm 3.4$ & $32.2 \pm 3.8$ & $32.3 \pm 3.2$ & 0.76 \\
\hline Hypertension (\%) & 66.4 & 61.7 & 69.6 & 0.38 \\
\hline Diabetes (\%) & 7.9 & 9.1 & 8.2 & 1.00 \\
\hline Number of MetS criteria (n) & $3(3-4)$ & $3(3-4)$ & $4(3-4)$ & $<0.01$ \\
\hline Apnea-hypopnea index (events/hour) & $19.6(7.1-39.0)$ & $5.0(2.6-8.8)$ & $34.7(25.0-54.3)$ & $<0.001$ \\
\hline Lowest oxygen saturation (\%) & $83(74-87)$ & $87(85-90)$ & $77(69-84)$ & $<0.001$ \\
\hline Total sleep time oxygen saturation $<90 \%(\%)$ & $3.0(0.2-10.0)$ & $0.3(0-1.8)$ & $8.9(2.7-24.5)$ & $<0.001$ \\
\hline Epworth sleepiness scale & $10(8-11)$ & $9(8-10)$ & $10(8-12)$ & 0.03 \\
\hline Excessive daytime sleepiness, $\uparrow$ n (\%) & $47(30.9)$ & $14(23.3)$ & $33(35.9)$ & 0.11 \\
\hline
\end{tabular}

*For comparisons between MetS patients with and without OSA. †Epworth sleepiness scale score $>10$.

MetS: Metabolic Syndrome. OSA: Obstructive Sleep Apnea. Variables with normal distribution are expressed as mean \pm SD. Variables with skewed distribution are presented as median (interquartile range).

doi:10.1371/journal.pone.0012065.t001 


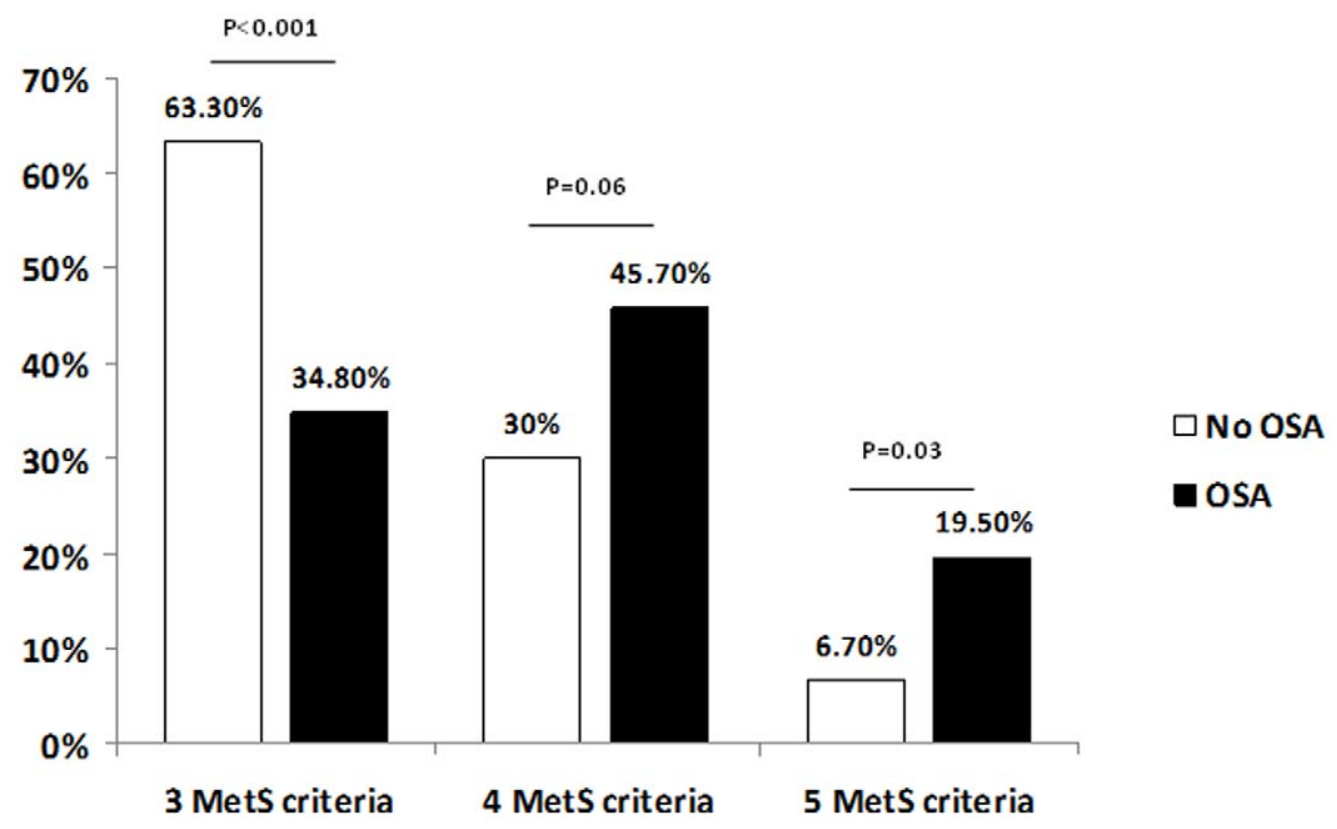

Figure 2. Rate of MetS criteria in patients with and without OSA. doi:10.1371/journal.pone.0012065.g002

\section{Discussion}

The present study showed that, in consecutive patients with MetS, unrecognized OSA is common and independently associated with biomarkers of metabolic dysfunction and systemic inflammation. Specifically, OSA was associated with two MetS criteria, triglycerides and glucose; and with three non-MetS cardiovascular risk factors, cholesterol/HDL ratio, uric acid, and G-reactive protein. These associations were not influenced by the presence or absence of excessive daytime sleepiness. Taken together, our data suggest that OSA may contribute to the metabolic and cardiovascular burden of patients with MetS.
Previous clinical studies of MetS failed to consider OSA as a potential confounding factor that contributes the cardiovascular risk [29,30]. Accordingly, the American Heart Association Scientific statement on MetS briefly discussed OSA and classified it as being of interest to "other fields of medicine," giving it the same attention given to cholesterol gallstones and lypodystrophies [1]. Conversely, the majority of previous investigations derived from the sleep community were intrinsically biased studying only patients referred for sleep studies [11-16]. Our study design allowed us to systematically examine the prevalence of unrecognized OSA in consecutive sample of patients with MetS. We found a $60.5 \%$ prevalence of OSA in MetS, even using conservative

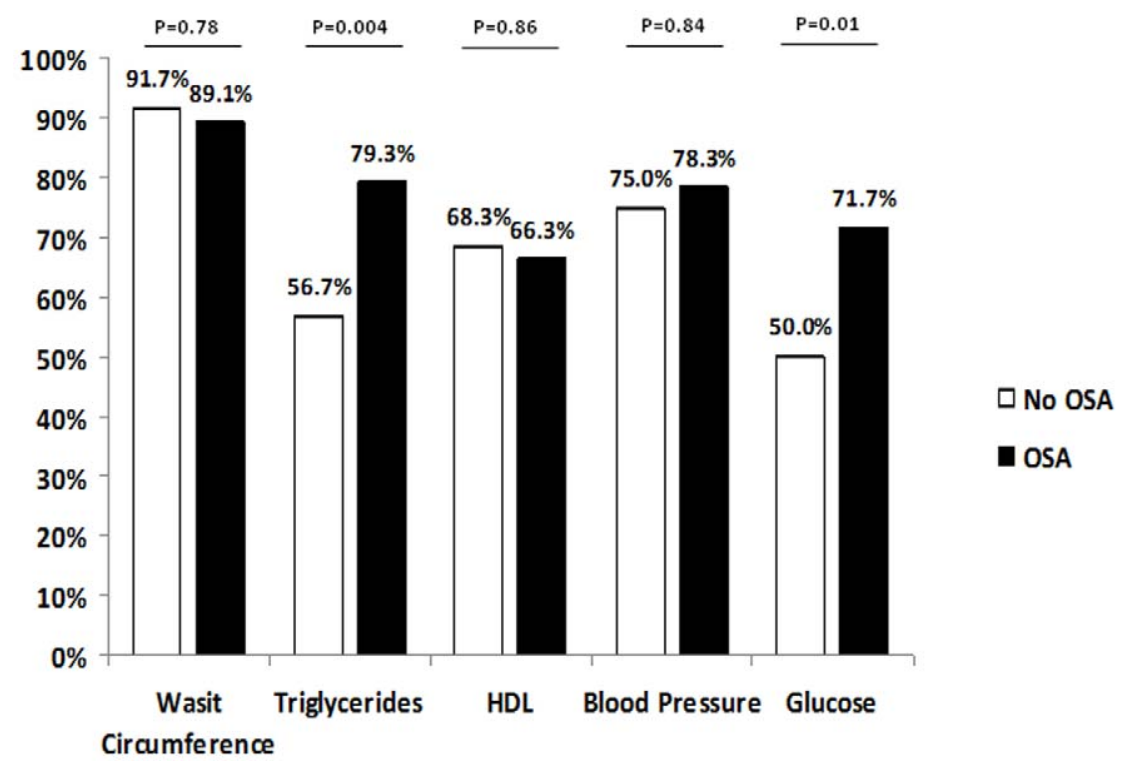

Figure 3. Frequency of each MetS criteria between groups (B). doi:10.1371/journal.pone.0012065.g003 
Table 2. Quantitative values of metabolic and inflammatory profile in Metabolic Syndrome patients with and without Obstructive Sleep Apnea.

\begin{tabular}{|c|c|c|c|c|}
\hline & Total Sample $(n=152)$ & No-OSA $(n=60)$ & OSA $(n=92)$ & $P$ value* \\
\hline \multicolumn{5}{|l|}{ Variables included in MetS criteria } \\
\hline Waist circumference $(\mathrm{cm})$ & $105.1 \pm 8.2$ & $104 \pm 9$ & $106 \pm 8$ & 0.16 \\
\hline Triglycerides (mg/dL) & $196 \pm 86$ & $170 \pm 81$ & $213 \pm 85$ & 0.002 \\
\hline HDL cholesterol (mg/DI) & $39(34-47)$ & $39(33-47)$ & $38(34-47)$ & 0.83 \\
\hline Systolic blood pressure (mm Hg) & $139 \pm 24$ & $134 \pm 22$ & $142 \pm 24$ & 0.03 \\
\hline Diastolic blood pressure $(\mathbf{m m ~ H g})$ & $84 \pm 14$ & $81 \pm 14$ & $86 \pm 15$ & 0.05 \\
\hline Fasting glucose (mg/dL) & $102 \pm 11$ & $98 \pm 10$ & $105 \pm 11$ & $<0.001$ \\
\hline \multicolumn{5}{|l|}{ Variables not included in MetS criteria } \\
\hline Total cholesterol (mg/dL) & $216 \pm 39$ & $204 \pm 32$ & $223 \pm 36$ & 0.003 \\
\hline Total cholesterol/HDL ratio & $5.5 \pm 1.4$ & $5.1 \pm 1.4$ & $5.7 \pm 1.5$ & 0.02 \\
\hline Triglycerides/HDL ratio & $5.1 \pm 2.9$ & $4.4 \pm 2.5$ & $5.6 \pm 2.9$ & 0.009 \\
\hline Uric acid (mg/dL) & $6.5 \pm 1.4$ & $5.8 \pm 1.3$ & $6.9 \pm 1.4$ & $<0.001$ \\
\hline C-reactive protein (mg/dL) & $3.0(2.0-4.0)$ & $2.6(1.3-3.2)$ & $3.9(2.7-4.4)$ & $<0.001$ \\
\hline
\end{tabular}

*For comparisons between patients with and without OSA.

doi:10.1371/journal.pone.0012065.t002

diagnostic OSA criteria (AHI $\geq 15$ events per hour of sleep). The prevalence of OSA observed in this study is in line with two previous reports from different groups (ranging from 68 to $87.5 \%$ ) that evaluated patients with MetS $[17,18]$. Consistently with our data, a recent report found a high prevalence of OSA $(\sim 86 \%)$ in obese patients with type 2 diabetes [31]. The high prevalence of OSA among our consecutive patients with MetS may be due to the shared feature of visceral obesity in both syndromes. In fact, visceral rather than the subcutaneous or total body fat predisposes to the development of OSA [32]. However, the effect of OSA on metabolic function in MetS, at least in terms of glucose control, has been shown to occur independently of waist circumference, a surrogate marker of visceral adiposity [18]. In the present larger study, which excluded patients treated with hypoglycemic and lipid-lowering medications, we show that the co-existence of OSA in patients with MetS is associated with increased glucose and triglycerides levels.

We have also shown that OSA is associated with non-MetS cardiovascular risk markers, including cholesterol/HDL ratio, uric acid, and C-reactive protein. These biomarkers have validated as indices of the cardiovascular risk. A large prospective study showed that a cholesterol/HDL ratio $>4.5$ is a better predictor of ischemic heart disease than total cholesterol, HDL cholesterol, or non-HDL cholesterol [33]. Uric acid, the catabolic end product of ATP, is associated with oxidative stress, inflammation, subclinical atherosclerosis, and an increased risk of cardiovascular events [3436]. Independent studies have shown that plasma uric acid is often elevated in subjects with the MetS and OSA $[37,38]$. Our study suggests that OSA has an additive effect on uric acid levels in patients with MetS. Finally, pro-inflammatory effects of MetS and

Table 3. Univariable and multivariable logistic regression analysis for the association between presence of OSA with variables included and not included in the MetS criteria.

\begin{tabular}{|c|c|c|c|}
\hline & Unadjusted odds ratio $(95 \% \mathrm{CI})$ & Adjusted odds ratio $(95 \% \mathrm{Cl}) *$ & Adjusted $P$ value \\
\hline \multicolumn{4}{|l|}{ Variables included in MetS criteria } \\
\hline Waist circumference criteria & $0.75(0.24-2.30)$ & $0.73(0.21-2.50)$ & 0.61 \\
\hline Triglycerides criteria & $2.94(1.43-6.02)$ & $3.26(1.47-7.21)$ & 0.004 \\
\hline HDL-C criteria & $0.91(0.46-1.83)$ & $0.87(0.42-1.80)$ & 0.71 \\
\hline Arterial blood pressure criteria & $1.20(0.56-2.58)$ & $1.02(0.45-2.32)$ & 0.96 \\
\hline Fasting glucose criteria & $2.54(1.29-5.01)$ & $2.31(1.12-4.80)$ & 0.02 \\
\hline \multicolumn{4}{|l|}{ Variables not included in MetS criteria } \\
\hline Total cholesterol/HDL ratio $\geq 4.5$ & $2.40(1.17-4.91)$ & $2.38(1.08-5.24)$ & 0.03 \\
\hline Triglycerides/HDL ratio $>3$ & $2.21(1.04-4.68)$ & $2.19(0.95-5.04)$ & 0.07 \\
\hline Uric Acid >7 mg/Dl & $4.18(1.78-9.82)$ & $4.19(1.70-10.35)$ & 0.002 \\
\hline C-reactive protein $>3 \mathrm{mg} / \mathrm{L}$ & $4.92(2.41-10.03)$ & $6.10(2.64-14.11)$ & $<0.001$ \\
\hline
\end{tabular}

*Adjusted for age, sex, race, body mass index and waist circumference (except for waist circumference criteria).

doi:10.1371/journal.pone.0012065.t003 
Table 4. Stepwise linear regression analysis for the association between markers of OSA severity and components of MetS and metabolic/inflammatory variables not included in the MetS criteria*.

\begin{tabular}{|c|c|c|c|c|}
\hline & Variables & Coefficient $(\beta)$ & 95\% Cl & $P$ value \\
\hline \multicolumn{5}{|c|}{ Variables included in MetS criteria } \\
\hline \multirow[t]{3}{*}{ Triglycerides } & Race & -14.530 & $-27.930-1.150$ & 0.03 \\
\hline & Body mass index & -5.670 & $-10.740-0.600$ & 0.03 \\
\hline & Minimum $\mathrm{O}_{2}$ saturation & -2.079 & $-4.120-0.040$ & 0.04 \\
\hline \multirow[t]{2}{*}{ Glucose } & Age & 0.315 & 0.1080 .522 & 0.003 \\
\hline & Apnea-hypopnea index & 0.081 & 0.0140 .148 & 0.02 \\
\hline \multicolumn{5}{|c|}{ Variables not included in MetS criteria } \\
\hline Cholesterol/HDL ratio & Apnea-hypopnea index & 0.013 & 0.0040 .022 & 0.006 \\
\hline \multirow[t]{2}{*}{ Uric Acid } & Sex & 0.845 & 0.3491 .342 & 0.001 \\
\hline & Apnea-hypopnea index & 0.013 & 0.0020 .024 & 0.02 \\
\hline \multirow[t]{3}{*}{ C-reactive protein } & Minimum $\mathrm{O}_{2}$ saturation & -0.042 & $-0.072-0.013$ & 0.005 \\
\hline & Sex & -0.632 & $-1.249-0.002$ & 0.04 \\
\hline & Age & 0.036 & 0.0030 .070 & 0.03 \\
\hline
\end{tabular}

*Variables used in the model: age, sex, race, body mass index, waist circumference and sleep parameters (apnea-hypopnea index, minimum $\mathrm{O}_{2}$ saturation during sleep and total sleep time below $90 \%)$.

doi:10.1371/journal.pone.0012065.t004

OSA were widely discussed in the literature and many independent studies reported that both MetS [26] and OSA [39] are independently associated with high C-reactive protein levels, which is a marker of cardiovascular inflammation. C-reactive protein adds clinically important prognostic information to the MetS [26]. Collectively, our study suggests that the severity of OSA

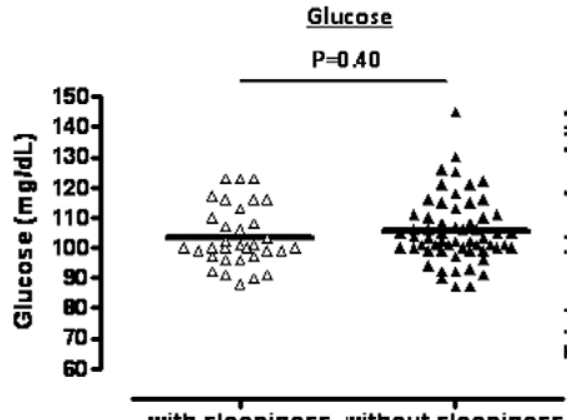

with sleepiness without sleepiness

Cholesterol/HDL ratio
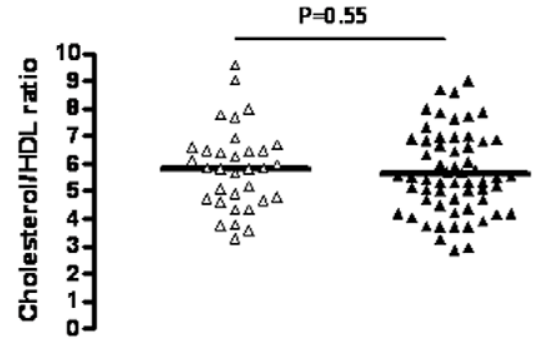

with sleepiness without sleepiness
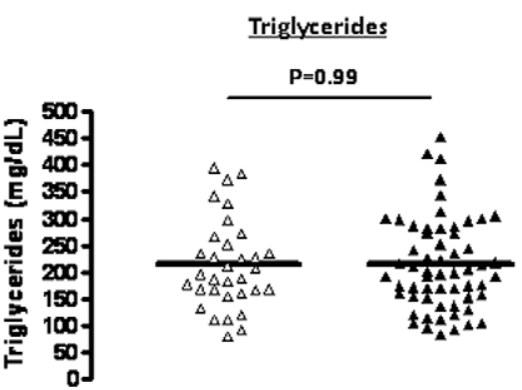

with sleepiness without sleepiness
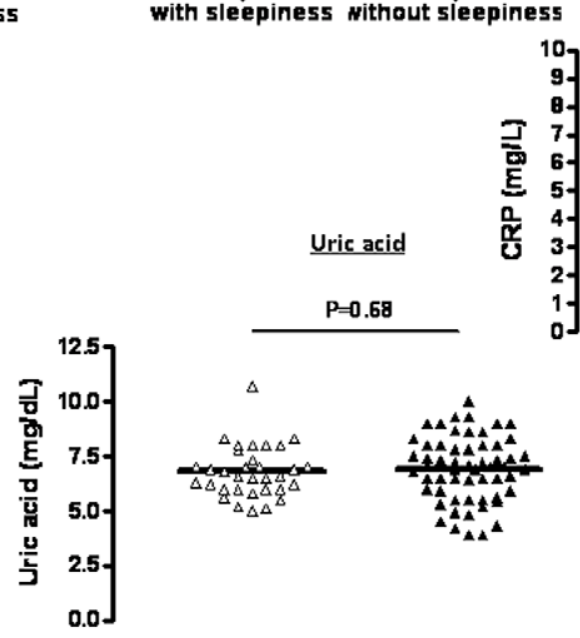

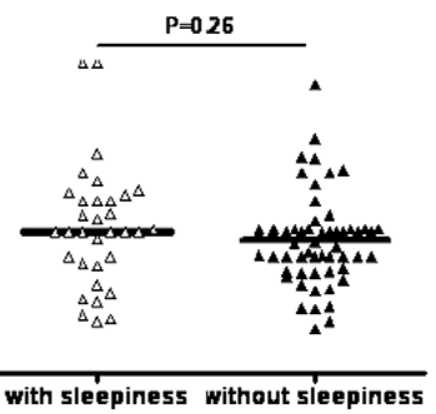

with sleepiness without sleepiness

C.reactive protein

Figure 4. Levels of glucose, triglycerides, cholesterol/HDL ratio, uric acid and C-reactive protein in patients with MetS and OSA according to the presence or absence of excessive daytime sleepiness.

doi:10.1371/journal.pone.0012065.g004 
is independently associated with unfavorable lipid profile, hyperuricemia and inflammation in consecutive patients with MetS.

The independent association of OSA with dyslipidemia and systemic inflammation observed in our study has biological basis. Intermittent hypoxia, the hallmark of OSA, causes dyslipidemia in mice by up-regulating hepatic lipid biosynthesis and lipoprotein secretion via hypoxia inducible factor 1 alpha $[9,10]$. Intermittent hypoxia also activates pro-inflammatory transcription factors such as nuclear factor kappa B that promote activation of various inflammatory cells with the downstream consequence of expression of pro-inflammatory mediators that may lead to endothelial dysfunction [40].

In the present study, we found that several metabolic and inflammatory markers associated with OSA were similar in patients with and without excessive daytime sleepiness. Other investigators have also shown that OSA is associated with markers of atherosclerosis [28] and mortality irrespective of daytime symptoms [41]. These collective results challenge the notion that only sleepy patients with OSA are at increased cardiovascular risk. OSA may not be suspected in non-sleepy persons, and, therefore, overlooked as a potential cardiovascular risk factor.

The main strength of our study is the study design, since consecutive subjects with MetS underwent full polysomnography, regardless of their sleep complaints. The main limitations are: First, the present study comprised a relatively small number of participants. The observation of a nonsignificant trend of an independent association between OSA and triglicerydes/HDL ratio, a marker of insulin resistance, may be due to the lack of power to detect this difference. Therefore, the impact of OSA in patients with MetS may be even greater. Second, our patients were middle-aged and without a history of coronary disease and stroke or intake of statins, fibrates and hypoglycemic drugs. Hence,

\section{References}

1. Grundy SM, Gleeman JI, Daniels SR, Donato KA, Eckel RH, et al.; American Heart Association; National Heart, Lung, and Blood Institute (2005) Diagnosis and management of the metabolic syndrome: an American Heart Association/ National Heart, Lung, and Blood Institute Scientific Statement. Circulation 112: 2735-2752. Erratum in: Circulation;112, e297.

2. Sundstrom J, Riserus U, Byberg L, Zethelius B, Lithell H, et al. (2006) Clinical value of the metabolic syndrome for long term prediction of total and cardiovascular mortality: prospective, population based cohort study. BMJ 332: 878-882.

3. Gami AS, Witt BJ, Howard DE, Erwin PJ, Gami LA, et al. (2007) Metabolic syndrome and risk of incident cardiovascular events and death: a systematic review and meta-analysis of longitudinal studies. J Am Coll Cardiol 49: 403414 .

4. Chen HJ, Bai CH, Yeh WT, Chiu HC, Pan WH (2006) Influence of metabolic syndrome and general obesity on the risk of ischemic stroke. Stroke 37: 1060-1064.

5. Ryan CM, Bradley TD (2005) Pathogenesis of obstructive sleep apnea. J Appl Physiol 99: 2440-2450.

6. Young T, Peppard PE, Gottlieb DJ (2002) Epidemiology of obstructive sleep apnea: a population health perspective. Am J Respir Crit Care Med 165: 1217-1239.

7. Peppard PE, Young T, Palta M, Skatrud J (2000) Prospective study of the association between sleep-disordered breathing and hypertension. N Engl J Med 342: $1378-1384$

8. Punjabi NM, Sorkin JD, Katzel LI, Goldberg AP, Schwartz AR, et al. (2002) Sleep-disordered breathing and insulin resistance in middle-aged and overweight men. Am J Respir Crit Care Med 165: 677-682.

9. Li J, Thorne LN, Punjabi NM, Sun CK, Schwartz AR, et al. (2005) Intermittent hypoxia induces hyperlipidemia in lean mice. Circ Res 97: 698-706.

10. Savransky V, Jun J, Li J, Nanayakkara A, Fonti S, Moser AB, et al. (2008) Dyslipidemia and atherosclerosis induced by chronic intermittent hypoxia are attenuated by deficiency of stearoyl coenzyme A desaturase. Circ Res 103: 1173-1180.

11. Coughlin SR, Mawdsley L, Mugarza JA, Calverley PM, Wilding JP (2004) Obstructive sleep apnoea is independently associated with an increased prevalence of metabolic syndrome. Eur Heart J 25: 735-741.

12. Gruber A, Horwood F, Sithole J, Ali NJ, Idris I (2006) Obstructive sleep apnoea is independently associated with the metabolic syndrome but not insulin resistance state. Cardiovasc Diabetol 5: 22. our results may not be applicable to other age groups, or patients with established cardiovascular disease. On the contrary, the exclusion of drugs that directly affect the metabolic and inflammatory profile in MetS may be an advantage for studying metabolic and pro-inflammatory effects of OSA. Third, we were unable to exclude patients on anti-hypertensive treatment, since more than $50 \%$ of patients were on medications, which could not be discontinued for ethical reasons. Finally, the cross sectional nature of the study does not prove cause-effect relationships between OSA and metabolic and inflammatory markers.

In conclusion, we have shown that OSA is highly common in patients with MetS. OSA is independently associated with increased prevalence and severity of hypertriglyceridemia and hyperglycemia, as well as with several other markers of metabolic and inflammatory dysregulation (cholesterol/HDL ratio, uric acid and C-reactive protein). Our data strongly suggest that patients with MetS need to be evaluated for OSA regardless of daytime sleepiness. We hypothesize that the presence of OSA can exacerbate MetS and further increase cardiovascular morbidity and mortality. Future interventional studies will demonstrate whether treatment of OSA will improve MetS and cardiovascular outcomes in these patients.

\section{Acknowledgments}

We thank Susheel Patil, MD, PhD for assistance with statistical analysis.

\section{Author Contributions}

Conceived and designed the experiments: LFD HL GLF. Performed the experiments: LFD CMN IT ETD MJA RF. Analyzed the data: LFD HLJJ EK VYP GLF. Contributed reagents/materials/analysis tools: HL CMN IT ETD MJA RF GN. Wrote the paper: LFD JJ GN EK VYP GLF.

13. Kono M, Tatsumi K, Saibara T, Nakamura A, Tanabe N, et al. (2007) Obstructive sleep apnea syndrome is associated with some components of metabolic syndrome. Chest 131: 1387-1392.

14. Sasanabe R, Banno K, Otake K, Hasegawa R, Usui K, et al. (2006) Metabolic syndrome in Japanese patients with obstructive sleep apnea syndrome. Hypertens Res 29: 315-322.

15. Parish JM, Adam T, Facchiano L (2007) Relationship of metabolic syndrome and obstructive sleep apnea. J Clin Sleep Med 13: 355-362.

16. Nock NL, Li L, Larkin EK, Patel SR, Redline S (2009) Empirical evidence for "syndrome Z": a hierarchical 5-factor model of the metabolic syndrome incorporating sleep disturbance measures. Sleep 32: 615-622.

17. Venkateswaran S, Shankar P (2007) The prevalence of syndrome Z (the interaction of obstructive sleep apnoea with the metabolic syndrome) in a teaching hospital in Singapore. Postgrad Med J 83: 329-331.

18. Drager LF, Queiroz EL, Lopes HF, Genta PR, Krieger EM, et al. (2009) Obstructive sleep apnea is highly prevalent and correlates with impaired glycemic control in consecutive patients with the metabolic syndrome. J Cardiometab Syndr 4: 89-95.

19. Chin K, Oga T, Takahashi K, Takegami M, Nakayama-Ashida Y, et al. (2010) Associations between obstructive sleep apnea, metabolic syndrome, and sleep duration, as measured with an actigraph, in an urban male working population in Japan. Sleep 33: 89-95.

20. Lam JC, Lam B, Lam CL, Fong D, Wang JK, et al. (2006) Obstructive sleep apnea and the metabolic syndrome in community-based Chinese adults in Hong Kong. Respir Med 100: 980-987.

21. Chobanian AV, Bakris GL, Black HR, Cushman WC, Green LA, et al.; Joint National Committee on Prevention, Detection, Evaluation, and Treatment of High Blood Pressure. National Heart, Lung, and Blood Institure; National High Blood Pressure Education Program Coordinating Committee. (2003) Seventh report of the Joint National Committee on Prevention, Detection, Evaluation, and Treatment of High Blood Pressure. Hypertension 42: 1206-1252.

22. Drager LF, Bortolotto LA, Lorenzi MC, Figueiredo AC, Krieger EM, et al. (2005) Early Signs of Atherosclerosis in Obstructive Sleep Apnea. Am J Respir Crit Care Med 172: 613-618.

23. Johns MW (1991) A new method for measuring daytime sleepiness: the Epworth sleepiness scale. Sleep 14: 540-545.

24. Linn S, Fulwood R, Rifkind B, Carroll M, Muesing R, et al. (1991) Serum total cholesterol: HDL cholesterol ratios in US white and black adults by selected 
demographic and socioeconomic variables (HANES II). Am J Public Health 81: 1038-1043.

25. McLaughlin T, Abbasi F, Cheal K, Chu J, Lamendola C, et al. (2003) Use of metabolic markers to identify overweight individuals who are insulin resistant. Ann Intern Med 139: 802-809.

26. Ridker PM, Buring JE, Cook NR, Rifai N (2003) C-reactive protein, the metabolic syndrome, and risk of incident cardiovascular events: an 8-year followup of 14719 initially healthy American women. Circulation 107: 391-397.

27. Lee K, Song YM, Sung J (2008) Which obesity indicators are better predictors of metabolic risk?: healthy twin study. Obesity (Silver Spring) 16: 834-840.

28. Drager LF, Bortolotto LA, Maki-Nunes C, Trombetta IC, Alves MJ, et al. (2010) The incremental role of obstructive sleep apnoea on markers of atherosclerosis in patients with metabolic syndrome. Atherosclerosis 208: 490-495.

29. Isomaa B, Almgren P, Tuomi T, Forsén B, Lahti K, et al. (2001) Cardiovascular morbidity and mortality associated with the metabolic syndrome. Diabetes Care 24: 683-689.

30. Arnlöv J, Ingelsson E, Sundström J, Lind L (2010) Impact of body mass index and the metabolic syndrome on the risk of cardiovascular disease and death in middle-aged men. Circulation 121: 230-236.

31. Foster GD, Sanders MH, Millman R, Zammit G, Borradaile KE, et al. (2009) Obstructive sleep apnea among obese patients with type 2 diabetes. Diabetes Care 32: 1017-1019.

32. Vgontzas AN, Papanicolaou DA, Bixler EO, Hopper K, Lotsikas A, et al. (2000) Sleep apnea and daytime sleepiness and fatigue: relation to visceral obesity, insulin resistance, and hypercytokinemia. J Clin Endocrinol Metab 85: 1151-1158.

33. Prospective Studies Collaboration, Lewington S, Whitlock G, Clarke R, Sherliker P, Emberson J, et al. (2007) Blood cholesterol and vascular mortality by age, sex, and blood pressure: a meta-analysis of individual data from 61 prospective studies with 55,000 vascular deaths. Lancet 370: 1829-1839. Erratum in: Lancet.2008;372:292.

34. Ruggiero C, Cherubini A, Miller E 3rd, Maggio M, Najjar SS, et al. (2007) Usefulness of uric acid to predict changes in C-reactive protein and interleukin-6 in 3-year period in Italians aged 21 to 98 years. Am J Cardiol 100: 115-121.

35. Santos RD, Nasir K, Orakzai R, Meneghelo RS, Carvalho JA, et al. (2007) Relation of uric acid levels to presence of coronary artery calcium detected by electron beam tomography in men free of symptomatic myocardial ischemia with versus without the metabolic syndrome. Am J Cardiol 99: 42-45.

36. Bos MJ, Koudstaal PJ, Hofman A, Witteman JCM, Breteker MMB (2006) Uric acid is a risk factor for myocardial infarction and stroke. Stroke 37: 1503-1507.

37. Tsouli SG, Liberopoulos EN, Mikhailidis DP, Athyros VG, Elisaf MS (2006) Elevated serum uric acid levels in metabolic syndrome: an active component or an innocent bystander? Metab Clin Exp 55: 1293-1301.

38. Steiropoulos P, Kotsianidis I, Nena E, Tsara V, Gounari E, et al. (2009) Longterm effect of continuous positive airway pressure therapy on inflammation markers of patients with obstructive sleep apnea syndrome. Sleep 32: 537-543.

39. Lui MM, Lam JC, Mak HK, Xu A, Ooi C, et al. (2009) C-reactive protein is associated with obstructive sleep apnea independent of visceral obesity. Chest 135: 950-956. Erratum in: Chest. 2009;135:1406.

40. Ryan S, Taylor CT, McNicholas WT (2005) Selective activation of inflammatory pathways by intermittent hypoxia in obstructive sleep apnea syndrome. Circulation 112: 2660-2667.

41. Young T, Finn L, Peppard PE, Szklo-Coxe M, Austin D, et al. (2008) Sleep disordered breathing and mortality: eighteen-year follow-up of the Wisconsin sleep cohort. Sleep 31: 1071-1078. 